\title{
PERIPHERAL CIRCULATION BY PHOTO-ELECTRIC RECORDING
}

\author{
BY \\ BERNARD LEIBEL
}

From the Buckston Browne Research Laboratory (Royal College of Surgeons) and Guy's Hospital

Received March 2, 1940

There is a growing tendency in modern medicine to rely on accessory methods and tests for clinical information, though nothing can really take the place of accurate observation and experience. It is the purpose of this paper to add yet another investigation to clinical science, but it is done to further the understanding of certain cardiovascular conditions and not to supplant the present methods of examination.

Numerous methods have been employed in the study of the peripheral circulation. In animal experiments the stromuhr is a familiar instrument, and by this means much interesting information has been obtained and many fundamental physiological facts have been established.

A more recent method of Rein (1928) involves the installation of a thermoelectric couple unit on the vessel wall. It consists of a pair of copper-constantin junctions between which is a heating element-the proximal at blood temperature and the distal at a higher temperature because of the warming effect of the heating element. This difference in temperature creates an electrical potential, which is recorded on a galvanometer, and the faster the blood flows past the point of heat application, the less heat it will acquire and the shorter will be the excursion of the galvanometer. Thus, after calibration, the deflection of the galvanometer will be a measure of the rate of blood flow. This method was improved upon by Herrick, Essex, Baldes, and Mann (1936), so that it is now applicable even to the coronary circulation.

Plethysmography has added much to the physiology of the circulation. In principle it depends upon the alteration of volume with changes in its blood content. Much apparatus has been devised for purposes of recording, at first of a simple type such as the Marey tambour or piston recorder, and later more refined optical and photographic methods. Goetz (1939) and Matthes (1935) transmitted the volumetric fluctuations to a graduated pipette or capillary tube, while Baldes and Corbeille (1928-9) and Bolton, Carmichael, and Stürup (1936) converted the volume changes to pressure changes by means of a water or membrane manometer. Sir Thomas Lewis has employed plethysmography to 
its best advantage, and to him we owe much of our knowledge concerning the physiology and pathology of the peripheral circulation. However, this method involves many technical difficulties, and the apparatus is bulky and inconvenient for routine or emergency investigation in the clinical wards. There is also a great deal of controversy over the interpretation of the results (Goetz, 1939), owing to possible errors inherent in the instrument itself and lack of detail in the records obtained.

\section{METHOD OF INVESTIGATION}

A more direct approach to the study of the peripheral circulation may be made by transillumination of the vascular bed, either by directly transmitted light or by reflected light that has penetrated the skin's surface. The intensity of the emergent beam will be found to fluctuate, paralleling in time and quantity the ebb and flow of blood circulating through the transilluminated tissue. These rhythmical changes are converted into electrical energy by a photo-electric cell. In turn, a capacity-coupled amplifier transforms the current so produced into potential energy which is recorded on an ordinary electrocardiograph.

Fig. 1 shows the actual apparatus, consisting of a standard model electro-

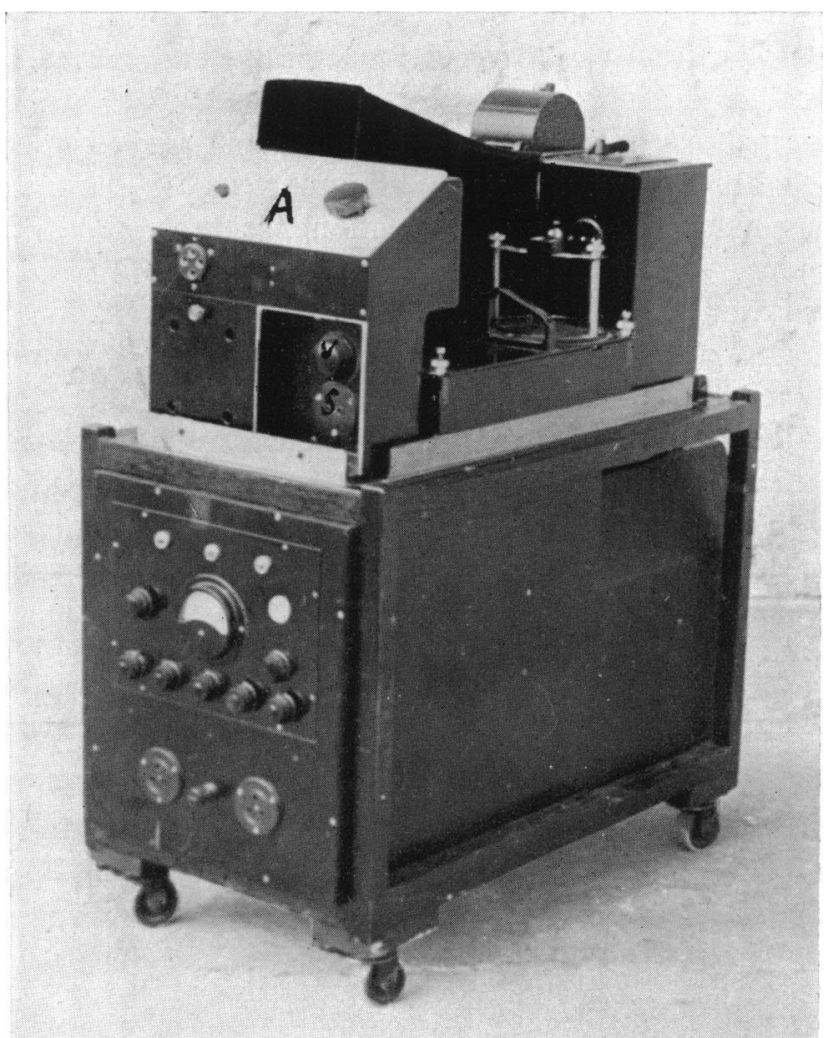

FIG. 1.-Combined electrocardiograph and tissue circulation recorder.

A is shown in more detail in Fig. 3, and the amplifier panel board (here shown in front is given in more detail in Fig. 6. 
cardiograph, amplifier, and battery, all mounted on a portable oak frame. It was found best to power the entire apparatus by a 12-volt battery, as this eliminated the need for long leads, avoided the possibility of a fluctuating and irregular mains supply, and enabled the entire apparatus to be shielded against electrical interference.

The unit for transillumination of the fingers or toes is shown in Fig. 2. The source of light is a focusing lamp with two thirds of the sphere silvered to provide a reflecting surface. The light is thus concentrated on the tissues and provides a maximum of light with a minimum of heat.

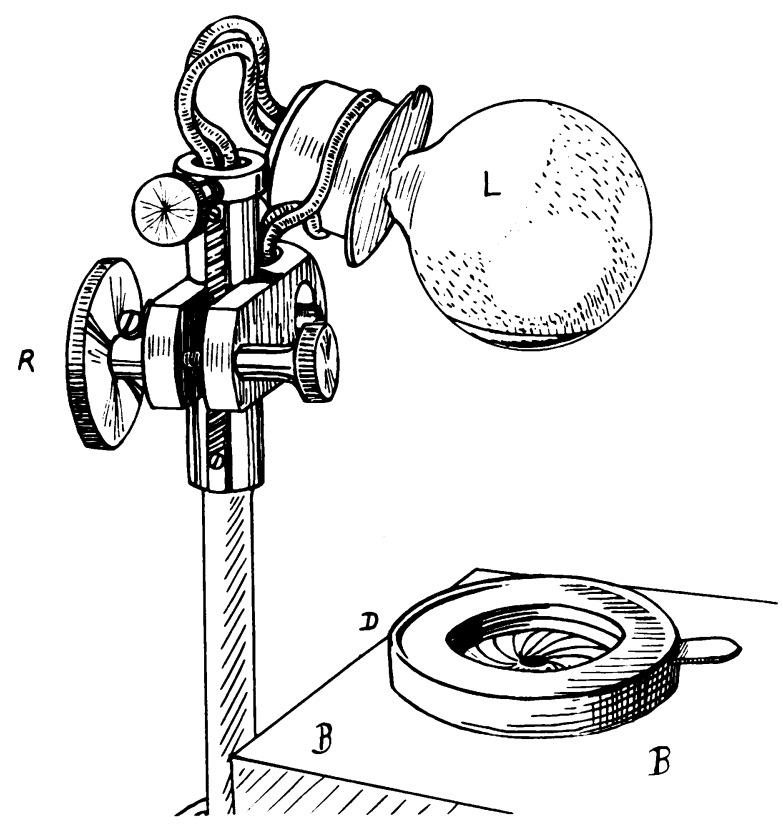

FIG. 2.-The photo-electric cell unit.

L, lamp ; R, ratchet device ; D, iris diaphragm ; and B, metal box housing the photoelectric cell.

The aperture in the iris diaphragm controls the amount of light that is allowed to fall on the photo-electric cell mounted beneath. The object to be transilluminated is placed over the aperture and the distance of the light source is regulated by the ratchet mounting device. The intensity of the light is adjusted by a variable resistance which is mounted in the electrocardiograph chassis (Fig. 3).

The Photo-electric Cell.-This is of the photo-emissive selenium type, No. PE 7B (B.T.H.). It is mounted by means of a four-pin holder on a wooden block, which in turn is fastened securely to the lid of the metal box. The inside of the container is painted black to minimize the possibility of extraneous light falling on the sensitive cell. The shielded lead from the cell, together with the flex lead to the lamp, is encased in rubber tubing to be conducted to the instrument proper.

It is of the utmost importance to realize that any abnormalities in this part 


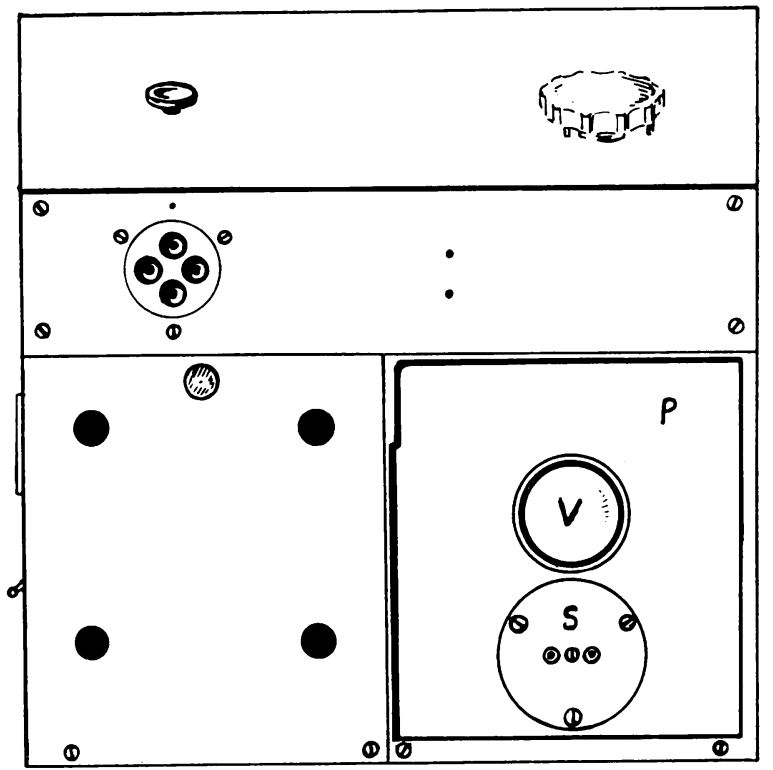

FIG. 3.-Panel mounting in electrocardiograph chassis. $\mathrm{P}$, panel ; V, variable resistance control ; and $\mathrm{S}$, lamp socket.

of the apparatus will be amplified several thousand times and result in extreme distortion of the subsequent records. Thus all precautions must be taken towards adequate insulation and shielding.

The Amplifier.-The actual circuit of the amplifier is presented in Fig. 4 (on the opposite page). Its components are standard materials and its construction involves no technical difficulties. However, the values throughout the circuit are very essential and must be strictly adhered to. While an amplifier cannot create new wave forms, it can distort those already present.

A few of the different complexes obtainable simply by varying the values in the amplifier circuit are shown in Fig. 5, and this emphasizes the need for maintaining the exact standards suggested. Previous methods employed by Hertzmann (1937) and by Matthes and Hauss (1938) further illustrate this point. It was thus necessary to search for a means of testing the accuracy of the wave-forms as they were translated by the amplifier. Since the rate, rhythm, and amplitude of an electrocardiogram coincides to a great extent with the wave forms of tissue circulation, it was decided to make this the criterion of accuracy of the amplifier unit. The method used was as follows : A normal electrocardiogram is taken. The amplifier is then introduced into the circuit and the string is accordingly tightened to compensate for the increased swing of the galvanometer. The artifact due to the momentum of the galvanometer string when swinging in a wide amplitude was thus avoided. The values in both the input and output circuits of the amplifier were then altered in the direction minimizing distortion. The circuit described here gives an almost identical image of the original cardiogram. 


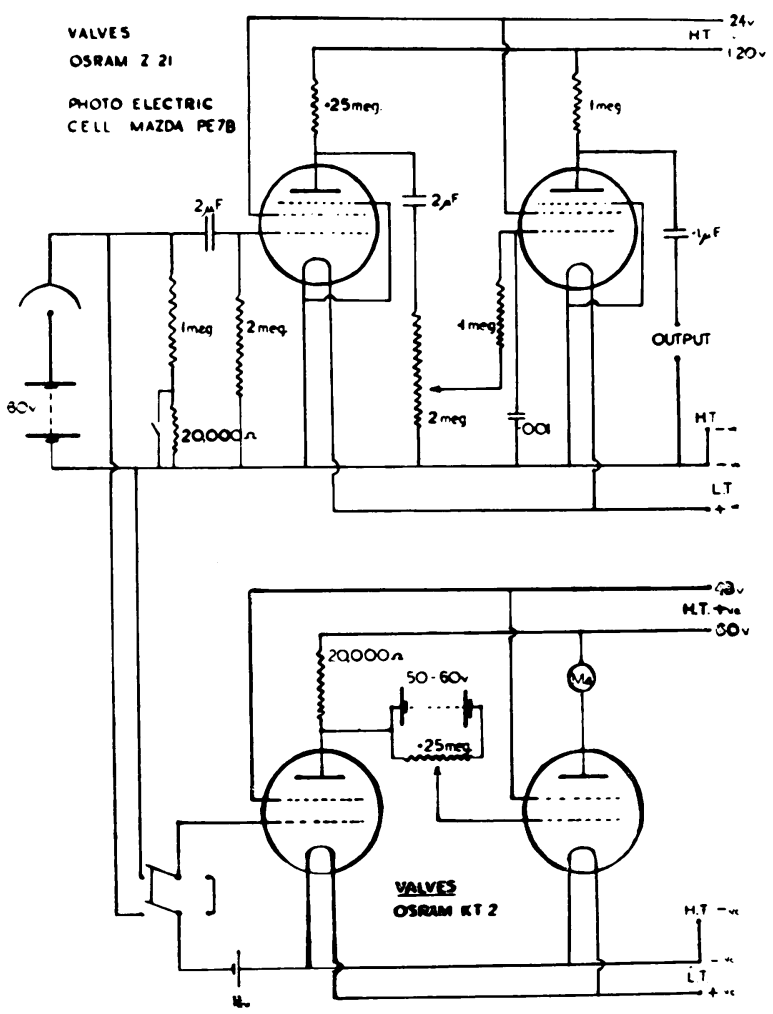

FIG. 4.-The combined electrical circuit of amplifier (above) and photometer (below). The values throughout the circuit are very essential and must be adhered to strictly.
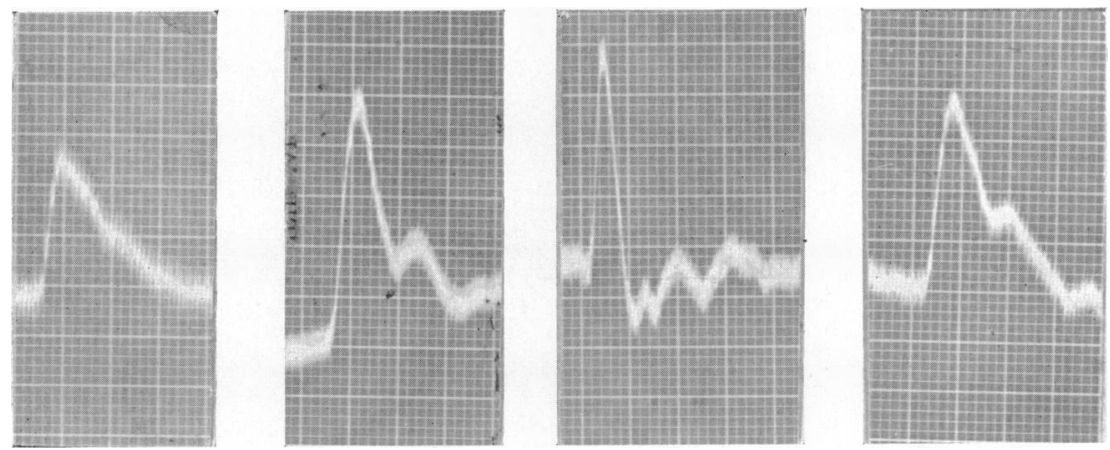

FIG. 5.-The wave-form distortion produced by altering the amplifier values.

The greater the intensity of the light transilluminating the vascular bed, the greater will be the resultant fluctuations and hence the amplitudes of the wave-forms recorded. The other factor controlling this feature of the wave form is the force of the heart beat. Thus, if one could standardize the amount of light actually transilluminating every vascular bed, one could interpret the amplitude of each complex in terms of heart force. Now in transilluminating, 
say, a finger-tip, the light has to pass through skin, fat, fascia, vessels, nerves, and bone. All of these, excepting the vessels with their rhythmical alteration in blood content, are constants throughout the period of record taking, but vary from individual to individual. Thus by constructing a direct current photometer recording on a milliammeter, the intensity of the light may be altered so that the same quantity of filtrate is obtained in every case. This amount was decided upon arbitrarily, but with the following provision. There is a tendency for the lamp to become quite warm, and owing to its proximity to the tissues to heat them in turn, so that a local vasodilatation results which is pure artifact. To reduce this difficulty, a focusing light described above was used, and before the standard quantity of light was chosen a series of tests was performed with a thermometer in position over the iris diaphragm. However, there have been occasional cases, especially those in which peripheral vascular disease was present, when an increased amount of light was necessary, and reflected light from a mirror had to be used in these to avoid local heating. The electrical circuit for this photometer unit has been shown in the lower half of Fig. 4. The arrangement of the various components of the amplifier panel board is indicated in Fig. 6.

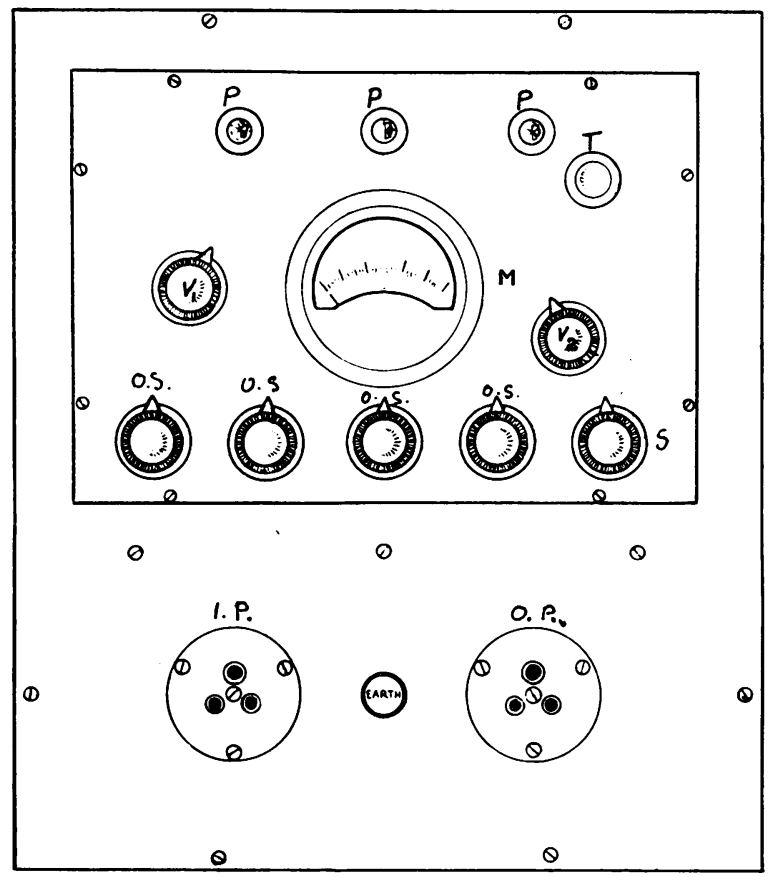

Fig. 6.-The amplifier panel board.

$\mathbf{P}$, pilot light ; $\mathbf{M}$, milliammeter ; $\mathrm{T}$, test button ; $\mathrm{V}_{\mathbf{1}}$, variable resistance photometer circuit ; $V_{2}$, variable resistance amplifier circuit ; I.P., input plug ; O.P., output plug ; S, shunt switch ; and O.S., on-off switches. 


\section{Method of taking the Records}

The procedure for taking records is first to carry out the light calibration. The photometer circuit is switched on and, by means of the variable resistance control, a constant current of one milliampere is employed. The light and photoelectric cell are then brought into the circuit with the tissue to be transilluminated in position. The intensity of the light, the position of the lamp, and the size of the aperture in the diaphragm are altered to suit the designated standard that is specific for each instrument. The photometer is then switched off and the amplifier turned on. The amplifier has in its circuit a standard cell system, similar to that employed for adjusting the tension of the galvanometer string in the electrocardiograph, and so the same amplification is always obtained. The lead from the amplifier is inserted into the socket for the cardiographic leads, and the procedure from here is the same as for an ordinary electrocardiogram. A protective shunt is incorporated in the amplifier unit to prevent excessive deflection of the galvanometer string during the preliminary adjustments.

\section{Results ObTAined}

Illustrations of the tissue circulation records obtained in normal subjects and in several pathological states follow. Each normal complex consists of a major systolic wave followed by a secondary diastolic wave (Fig. 7 A). In a continuous record sinus arhythmia may be observed, and this is apparent, not only as a change in the rate of the heart, but also as a corresponding change in the force of the heart, which is manifested by alteration in the height of the systolic waves. This is illustrated in Fig. 10 also.

Fig. 7 B shows the effect of excitement and moderate exercise on the normal

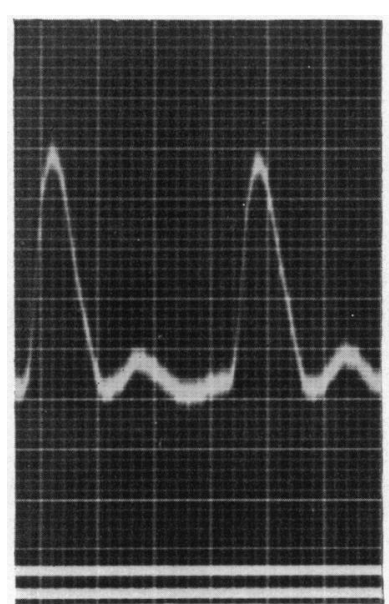

A

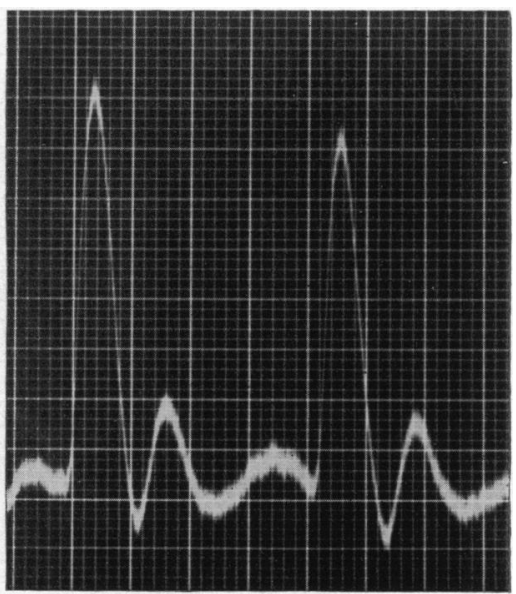

B

FIG. 7.-(A) Normal tissue circulation record. (B) Record after exercise. 
wave-forms. The heights of both the systolic and diastolic waves are increased, and a third wave makes its appearance in the presystolic period.

Fig. $8 \mathrm{~A}$ was obtained after holding the breath for forty-five seconds. It is interesting to see that the diastolic wave still maintains its force in spite of a weakened systolic beat.

Vasodilatation had a marked effect on the tissue circulation record. Amyl nitrite was inhaled and after three minutes Fig. 8 B was taken. There was a great increase in the wave excursions, especially the diastolic one, as there was after exercise.

A normal hand was bathed in water at a temperature of $60^{\circ} \mathrm{C}$. and Fig. $8 \mathrm{C}$ taken. The vasodilatation was not nearly so marked as in the previous record.

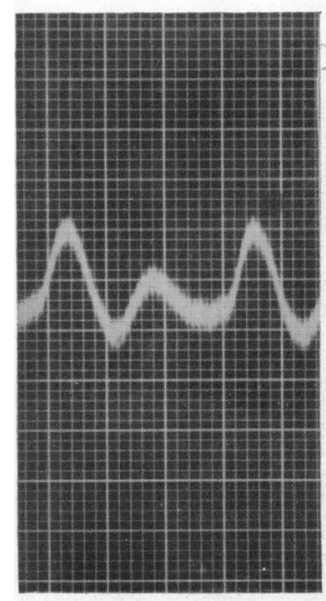

A

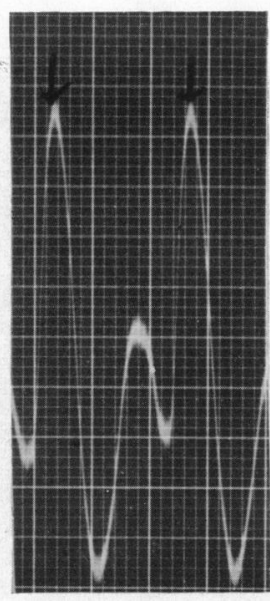

B

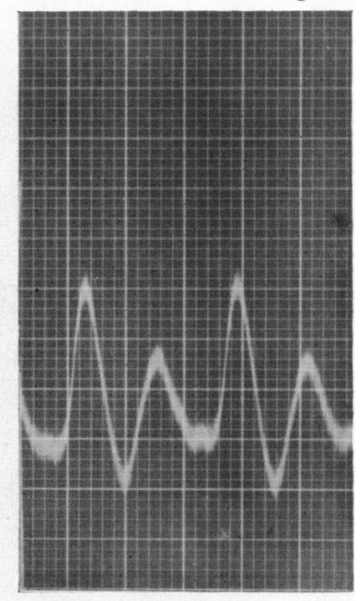

C

Fig. 9.-(A) Tissue circulation record in asphyxia. (B) Record after amyl nitrite. (C) Record after vasodilatation due to application of local heat.

Pulsus Bigeminus, Pulsus Alternans, and Pulsus Paradoxus.-Several varieties of pulse rhythm have been recorded. A curve of a typical pulsus bigeminus,

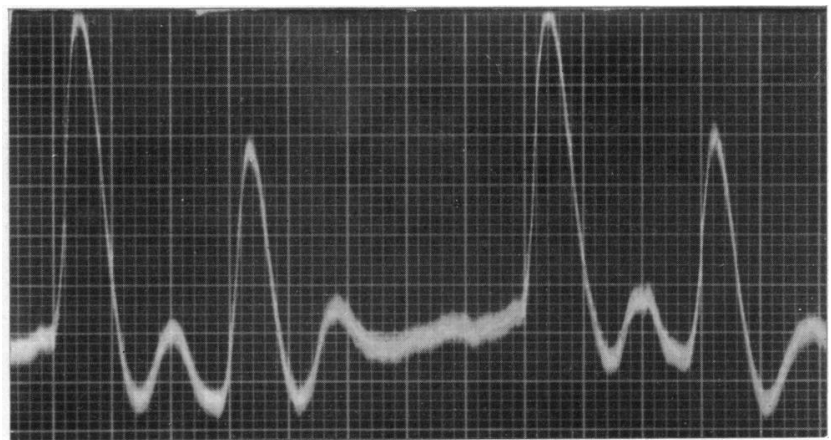

FIG. 9.-Tissue circulation record of pulsus bigeminus. 
from a case of digitalis poisoning, shows that the normal beat and extrasystole are coupled and that the extrasystole is a weaker beat (Fig. 9).

An early pulsus alternans is seen in Fig. 10, taken from a case of acute

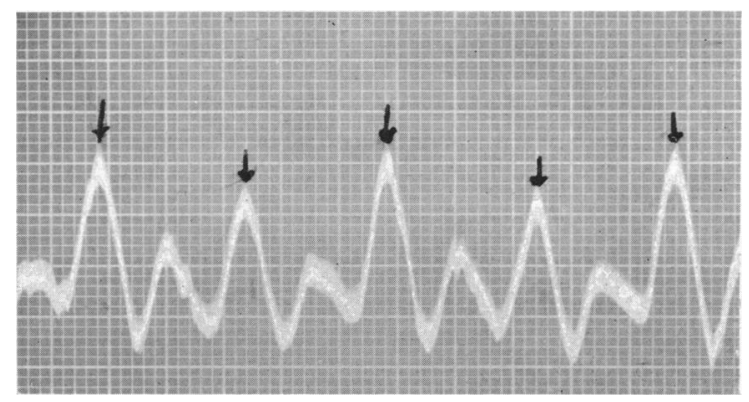

FIG. 10.-Tissue circulation record of pulsus alternans.

rheumatic heart disease. Actually in this record the weaker beat is not linked with the succeeding stronger one, but the variation in force between the beats is only $25 \mathrm{~mm}$. mercury.

Pulsus paradoxus from a case of constrictive pericarditis is shown in Fig. 11 ;

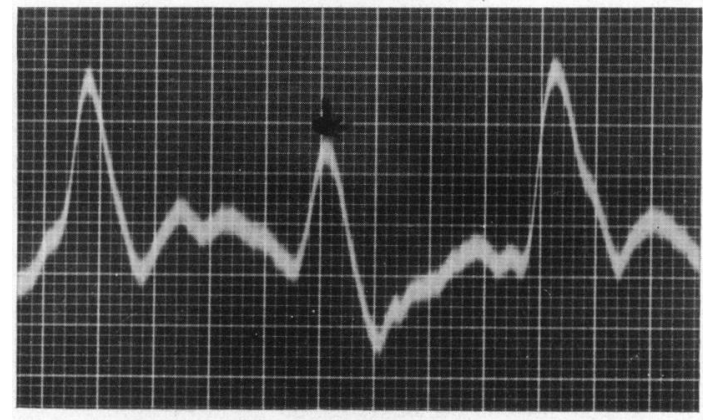

FIG. 11.-Tissue circulation record of pulsus paradoxus.

the weak beat always coincided with the height of inspiration. This is evidence of a constrictive band of fibrous tissue at the base of the heart interfering with its adequate filling during this phase of respiration.

Aortic Regurgitation.-A typical Corrigan or water-hammer pulse is illustrated in Fig. 12, taken from a patient with aortic regurgitation and an aneurysm. The mathematical equation for this wave-form is-

$$
\frac{d^{2} \log y}{d x^{2}}=1-y \quad \text { where } \quad \mathrm{Y} \max =2 .
$$

It is hoped that in a subsequent paper formulæ may be evolved for the other records, by which it may be possible to estimate the relative efficiency at which the heart is performing in the different lesions affecting it. Noteworthy in this record is the presence of sinus arhythmia affecting heart force as well as rate. 


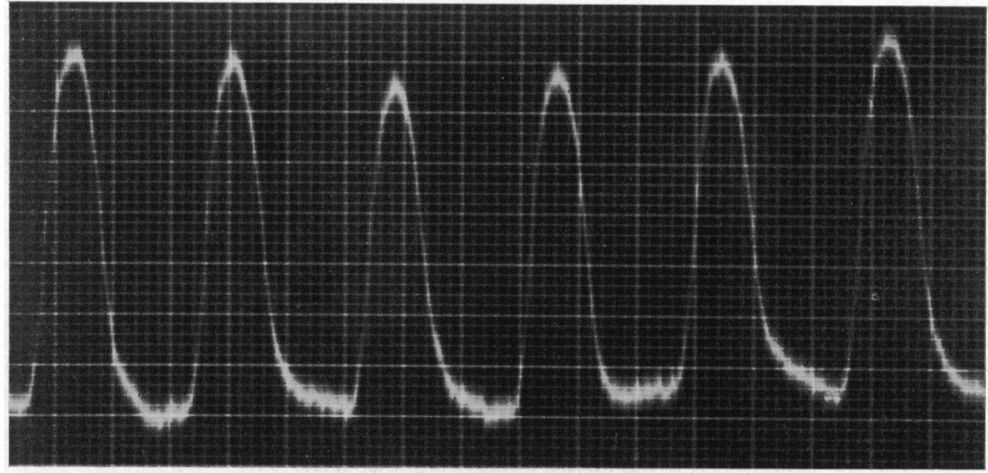

FIG. 12.-Tissue circulation record of a Corrigan pulse.

Auricular Fibrillation.-Fig. 13 is from a patient with auricular fibrillation ; the inconstant heart force is observed as well as the irregular rate. The longer

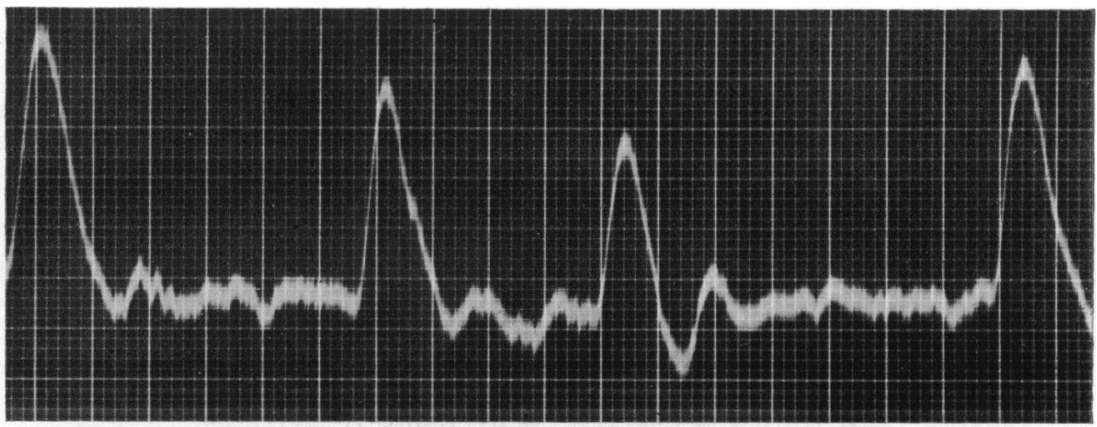

FIG. 13.-Tissue circulation record of auricular fibrillation.

the interval, the stronger the subsequent beat. This patient had a pulse deficiency at the radial artery of 20 beats per minute, but all the beats were readily picked up in the tissue circulation record by transilluminating the thumb.

Myocardial Failure.-In cases of myocardial degeneration with congestive failure, records such as Fig. 14 are obtained. Here there is normal rate and rhythm, but irregularity in the heart force and absence of a diastolic wave. This absence is seen again in Fig. 15, from a patient admitted in a state of collapse, with a diagnosis of coronary thrombosis.

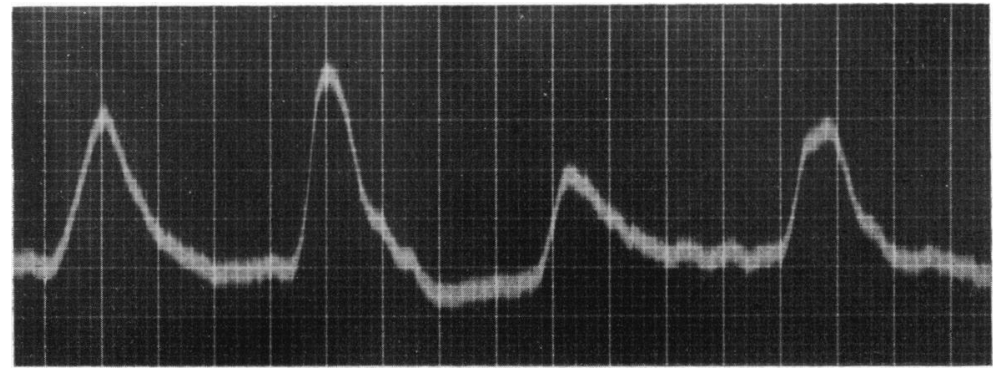

FIG. 14.-Tissue circulation/record from a case of congestive failure. 


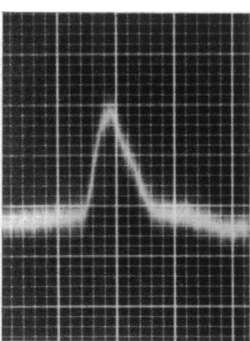

A

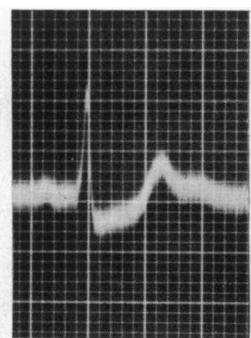

I

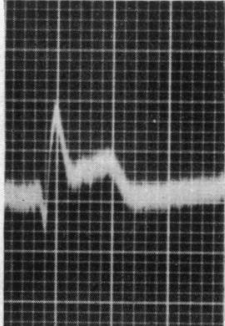

II

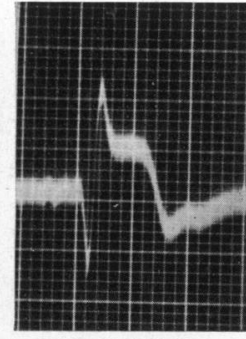

III

Fig. 15.-Tissue circulation record from a patient with coronary thrombosis (A) and leads I, II, and III of the electrocardiogram.

Fig. 16 is a tissue circulation record (A) and electrocardiogram of a patient with bradycardia due to heart block. The pulse was slow and sustained. His record shows a plateau-topped systolic impulse, but no diastolic wave.

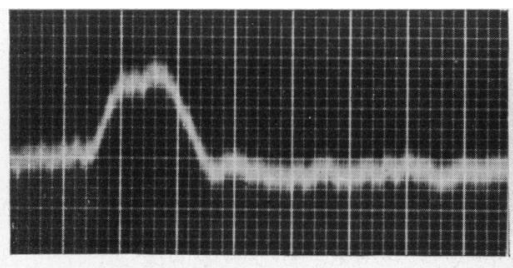

A

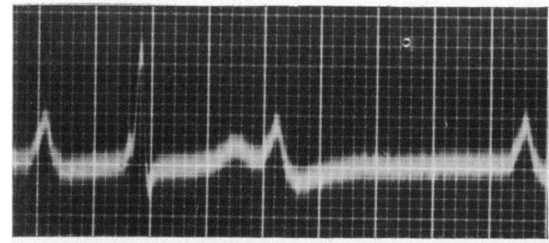

II

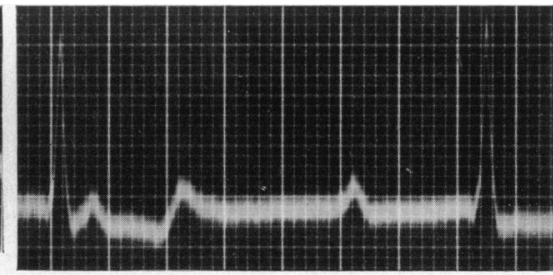

I

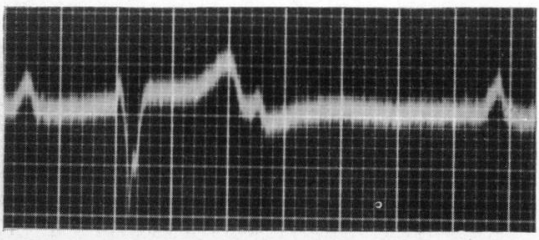

III

FIG. 16.-Tissue circulation record and leads I, II, and III of the electrocardiogram from a patient with complete heart block.

Peripheral Vascular Disease.-Fig. 17, obtained by transilluminating a finger, the tip of the left hallux, and the right hallux, illustrates the different states of tissue circulation in a man who suffered from obliterative peripheral vascular disease. There were no symptoms in the arms, and the left leg was considered normal, but the right foot was in a state of early dry gangrene. Comparison of the records shows that the left foot was only just on the threshold of normality and suggests that unless an adequate regime of treatment should be instituted, it too would become gangrenous.

The "Pavaex" glass boot has been recommended as an aid to establishing some collateral circulation. Fig. $18 \mathrm{~A}$ is a record from the base of a toe that 


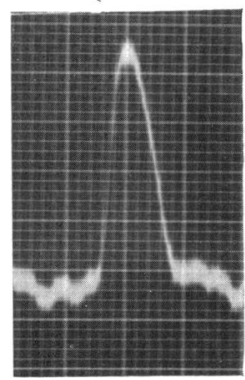

A

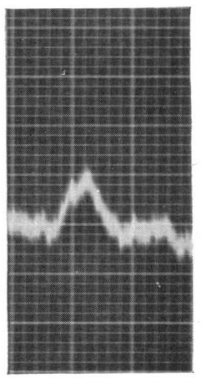

B

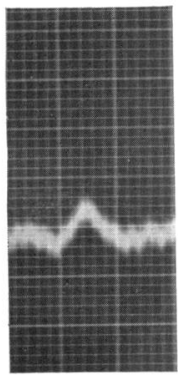

C

FIG. 17.-Tissue circulation record from a patient with peripheral vascular disease.

(A) From finger. (B) From left hallux. (C) From right hallux.

was involved in senile gangrene. The boot was applied for a period of three hours and Fig. $18 \mathrm{~B}$ indicates the state of the circulation two and a half hours after its installation, and Fig. $18 \mathrm{C}$ six hours after it had been removed. Fifteen hours later Fig. $18 \mathrm{D}$ was taken and shows that the improvement was not maintained.

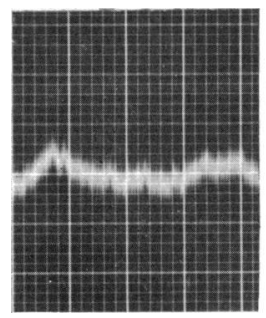

A

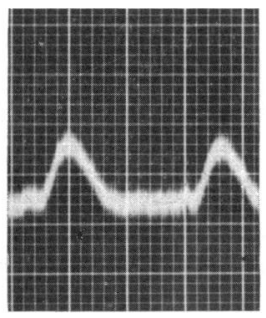

B

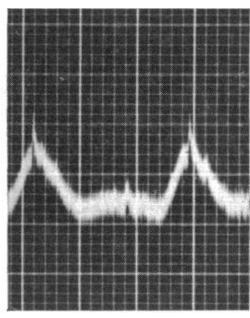

C

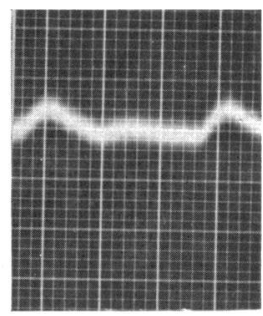

D

Fig. 18.-Records showing the effect of the "Pavaex glass boot" in producing temporary improvement in the circulation.

(A) Before treatment ; (B) during treatment ; (C) six hours after ; and (D) fifteen hours after treatment.

Measurement of Pulse Velocity.-By superimposing a normal electrocardiogram on a tissue circulation record, one can accurately time the interval between the electrical response of the apex beat and the appearance of the pulse in the tissues. This is done by joining the amplifier lead with that from the electrodes on the limbs, and thus a wave-form is obtained which is the resultant of the two sets of records ; an example is shown in Fig. 19. While the detail of these forms is now masked, the apex of the $R$ wave of the QRS complex is clearly visible, as is the apex (A) of the systolic wave of the circulation record. This $\mathrm{R}-\mathrm{A}$ interval can be measured in the same way as one would measure a P-R interval in the cardiogram. 


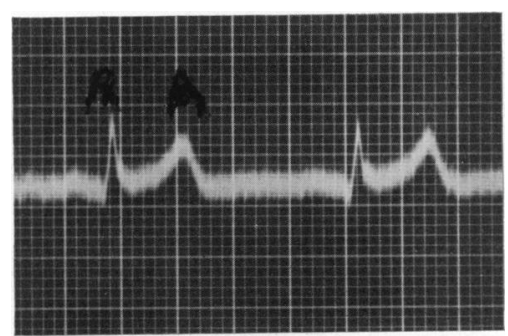

FIG. 19.-Record showing the time of the impulse conduction from apex to tissues.

It is hoped that in the near future it will be possible to publish separate papers with clinical details on some of the records that have been taken. In the meantime, these records have been presented to illustrate the possibilities of the instrument described and to serve as a guide to others who may carry out their own investigations.

\section{Summary AND CONCLUSIONS}

1. An instrument for recording vascular changes in the tissues of the extremities and of the body surface has been described. Calibration has been made possible by the introduction of a photometer circuit; results are therefore both qualitative and quantitative. In addition an accurate estimation of the pulse velocity may be made by superimposing the electrocardiogram on the tissue circulation record.

2. The amplifier circuit described is relatively free from electrical distortion and mechanical and physical artifact have been controlled by adequate insulation. The instrument is self-contained and readily portable, and records may be taken without any previous preparation of the patient.

3. A series of typical records has been presented and briefly described. Some of these illustrate physiological reactions to different stimuli, such as heat, excitement, asphyxia, and vasodilatation after amyl nitrite inhalation. The remainder of the records were obtained from cases of certain cardiovascular conditions. Examples of pulsus bigeminus, pulsus paradoxus, pulsus alternans, and the Corrigan pulse have been illustrated. Comparative estimation of the circulation in each of the limbs of a patient suffering from peripheral vascular disease has been included and the effect of the "Pavaex glass boot" has been demonstrated.

The author would like to thank the Royal College of Surgeons of England and its Director of Research for facilities placed at his disposal, and Sir Arthur Keith, Master of the Buckston Browne Research Farm, who has been a constant source of inspiration and encouragement. He would also like to thank Drs. E. P. Poulton, Maurice Campbell, and R. T. Grant for so generously providing cases for observation at Guy's Hospital.

Miss Mary Barclay Smith and Dr. C. Colbeck have been very helpful with illustrations and photographs, and Mr. F. Watson, senior technician at the laboratory, has been of great assistance throughout this work. 


\section{REFERENCES}

Baldes, E. J., and Corbeille, C. (1928-29). Proc. Soc. exp. Biol. N.Y., 26, 711.

Bolton, B., Carmichael, E. A., and Stürup, G. (1936). J. Physiol., 86, 83.

Goetz, R. H. (1940). Brit. J. Surg., 27, 506.

Essex, H. E., Herrick, J. F., Baldes, E. S., and Mann, F. C. (1936). Amer. J. Physiol., 117, 271.

Hertzman, A. B. (1937). Proc. Soc. exp. Biol. N.Y., 37, 529.

Matthes, K., and Hauss, W. (1938). Klin. Wschr., 17, 1211.

Matthes, K. (1935). Arch. exp. Path. Pharm., 179, 698.

Rein, H. (1928). Z. Biol., 87, 394. 